University of Wollongong

Research Online

Faculty of Science, Medicine and Health -

Papers: part A

Faculty of Science, Medicine and Health

$1-1-2005$

\title{
Systemic levels of interleukin- 6 and matrix metalloproteinase-9 in patients with multiple myeloma may be useful as prognostic indexes of bone disease
}

\author{
Aikaterini Sfiridaki \\ Venizelion General Hospital Heraklion \\ Spyridon Miyakis \\ University of Athens, smiyakis@uow.edu.au \\ George Tsirakis \\ University Hospital of Heraklion \\ Athanasios Alegakis \\ Medical School of Crete \\ Andreas M. Passam \\ Venizelion General Hospital Heraklion
}

See next page for additional authors

Follow this and additional works at: https://ro.uow.edu.au/smhpapers

Part of the Medicine and Health Sciences Commons, and the Social and Behavioral Sciences

\section{Commons}

\section{Recommended Citation}

Sfiridaki, Aikaterini; Miyakis, Spyridon; Tsirakis, George; Alegakis, Athanasios; Passam, Andreas M.; Kandidaki, Ermioni; Margioris, Andrerw N.; and Alexandrakis, Michael, "Systemic levels of interleukin-6 and matrix metalloproteinase-9 in patients with multiple myeloma may be useful as prognostic indexes of bone disease" (2005). Faculty of Science, Medicine and Health - Papers: part A. 210.

https://ro.uow.edu.au/smhpapers/210

Research Online is the open access institutional repository for the University of Wollongong. For further information contact the UOW Library: research-pubs@uow.edu.au 


\title{
Systemic levels of interleukin- 6 and matrix metalloproteinase- 9 in patients with multiple myeloma may be useful as prognostic indexes of bone disease
}

\begin{abstract}
Multiple myeloma is characterized by accelerated production of the proteolytic enzyme matrix metalloproteinase (MMP)-9. We hypothesized that myeloma- produced MMP-9 may influence the rate of bone turnover in a paracrine manner. Thus, we examined the correlations of MMP-9 levels, disease severity, and bone turnover rate as evaluated by markers of bone formation and resorption. Thirty-seven newly diagnosed multiple myeloma patients (nine of Durie-Salmon stage I, 12 of stage II and 16 of stage III) and 12 age-matched controls were studied. Serum MMP-9 levels were significantly higher at stage II compared to stage I (188.78"91.27 vs. $59.25 " 33.09 \mathrm{ng} / \mathrm{mL}, \mathrm{p}-0.004)$. Additionally, free urine pyridinolines (F-Pyd), free urine deoxy-pyridinolines (F-Dpd) and urine N-telopeptide fragment (NTx) were elevated, their level correlating with disease stage ( $\mathrm{p}-0.001, \mathrm{p}-0.03, \mathrm{p}-0.001$, respectively), as were bone marrow infiltration and serum interleukin- 6 (IL-6) levels ( $p-0.0001, p-0.01$, respectively). MMP-9 levels were lower in patients compared with controls ( $p-0.001)$, whereas IL- 6 and bone resorption marker levels were higher in patients than in controls ( $p-0.001$ in all cases). Significant correlation was found between infiltration, MMP-9, free urine pyd, free urine dpd and NTx for each stage of the disease (p-0.03, p-0.003, p-0.002, p- 0.003 and p-0.001, respectively). Levels of MMP-9 and of IL-6 in multiple myeloma correlate well with bone turnover rate and may be useful in disease evaluation.
\end{abstract}

\section{Keywords}

useful, prognostic, indexes, bone, disease, systemic, may, be, myeloma, levels, multiple, patients, 9 , metalloproteinase, matrix, 6 , interleukin

\section{Disciplines}

Medicine and Health Sciences | Social and Behavioral Sciences

\section{Publication Details}

Sfiridaki, A., Miyakis, S., Tsirakis, G., Alegakis, A., Passam, A. M., Kandidaki, E., Margioris, A. N. \& Alexandrakis, M. (2005). Systemic levels of interleukin- 6 and matrix metalloproteinase- 9 in patients with multiple myeloma may be useful as prognostic indexes of bone disease. Clinical Chemical Laboratory Medicine, 43 (9), 934-938.

\section{Authors}

Aikaterini Sfiridaki, Spyridon Miyakis, George Tsirakis, Athanasios Alegakis, Andreas M. Passam, Ermioni Kandidaki, Andrerw N. Margioris, and Michael Alexandrakis 


\section{Systemic levels of interleukin-6 and matrix metalloproteinase-9 in patients with multiple myeloma may be useful as prognostic indexes of bone disease}

\author{
Aikaterini Sfiridaki', Spiros Miyakis², George \\ Tsirakis ${ }^{3}$, Athanassios Alegakis ${ }^{4}$, Andreas M. \\ Passam ${ }^{1}$, Ermioni Kandidaki ${ }^{1}$, Andrew N. \\ Margioris $^{5}$ and Michael G. Alexandrakis ${ }^{3, *}$ \\ ${ }^{1}$ Blood Bank Center, Venizelion General Hospital \\ Heraklion, Crete, Greece \\ ${ }^{2}$ Third Department of Medicine, University of \\ Athens, Greece \\ ${ }^{3}$ Department of Haematology, University Hospital of \\ Heraklion, Crete, Greece \\ ${ }^{4}$ Department of Biostatistics, \\ ${ }^{5}$ Department of Clinical Chemistry-Biochemistry, \\ School of Medicine, University of Crete, Heraklion, \\ Greece
}

\begin{abstract}
Multiple myeloma is characterized by accelerated production of the proteolytic enzyme matrix metalloproteinase (MMP)-9. We hypothesized that myeloma-produced MMP-9 may influence the rate of bone turnover in a paracrine manner. Thus, we examined the correlations of MMP-9 levels, disease severity, and bone turnover rate as evaluated by markers of bone formation and resorption.

Thirty-seven newly diagnosed multiple myeloma patients (nine of Durie-Salmon stage I, 12 of stage II and 16 of stage III) and 12 age-matched controls were studied. Serum MMP-9 levels were significantly higher at stage II compared to stage I (188.78 \pm 91.27 vs. $59.25 \pm 33.09 \mathrm{ng} / \mathrm{mL}, \mathrm{p}<0.004)$. Additionally, free urine pyridinolines (F-Pyd), free urine deoxy-pyridinolines (F-Dpd) and urine $\mathrm{N}$-telopeptide fragment (NTx) were elevated, their level correlating with disease stage ( $p<0.001, p<0.03, p<0.001$, respectively), as were bone marrow infiltration and serum interleukin-6 (IL-6) levels $(p<0.0001, p<0.01$, respectively). MMP-9 levels were lower in patients compared with controls ( $p<0.001)$, whereas IL-6 and bone resorption marker levels were higher in patients than in controls ( $p<0.001$ in all cases). Significant correlation was found between infiltration, MMP-9, free urine pyd, free urine dpd and NTx for each stage of the disease $(\mathrm{p}<0.03, \mathrm{p}<0.003, \mathrm{p}<0.002, \mathrm{p}<0.003$ and $\mathrm{p}<0.001$, respectively). Levels of MMP-9 and of IL- 6 in multiple

\footnotetext{
*Corresponding author: Michael G. Alexandrakis, Assistant Professor of Medicine, Department of Haematology, University Hospital of Heraklion, P.O. Box 1352, Heraklion, Crete, Greece

Phone: +30-28-1039-2426, Fax: +30-28-1024-4883,

E-mail: freda@med.uoc.gr
}

myeloma correlate well with bone turnover rate and may be useful in disease evaluation.

Keywords: matrix metalloproteinases; multiple myeloma; N-telopeptide.

\section{Introduction}

Multiple myeloma (MM) is a malignant monoclonal proliferation of bone marrow plasma cells, with debilitating skeletal involvement $(1,2)$. Skeletal morbidity in MM correlates well with the degree of plasma cell infiltration $(3,4)$. The progression of the disease involves the production by the tumor cells of a specific class of extracellular matrix-degrading metalloenzymes, including matrix metalloproteinase (MMP)-9, a 92-kDa type IV collagenase $(5,6)$. MMP-9 degrades collagen IV, the major constituent of basement membrane, and this appears an essential step for cellular invasion and tumor progression (7-9). The pathogenesis of myeloma-induced bone disease is associated with accelerated bone turnover, leading to an imbalance between bone formation and resorption (10). MMP-9 is predominantly expressed in cells of macrophage lineage, such as osteoclasts that use it to hydrolyze bone collagen type I $(11,12)$. It is postulated that myeloma cell-derived MMP-9 - produced in the vicinity of bones - may enhance organic bone matrix absorption in a direct, paracrine fashion $(7,13)$. Malignant plasma cell infiltration has been shown to be directly responsible for the increased resorptive activity and abnormal remodeling, either by locally produced autocrine or paracrine osteoclast-activating factors or by direct cell to cell contact (1). Furthermore, the production of MMP-9 by myeloma cells could be accelerated locally by cytokines, chemokines and growth factors produced by bone cells, aggravating, thus, the invasive behavior of the malignant cells, by the establishment of a local self-accelerating cycle (14). Indeed, bone marrow endothelial cells were found to enhance MMP-9 production in MM, indicating that endothelial cells accelerate MM cell invasion via MMP-9 secretion $(7,13,15)$. Bone formation and resorption markers represent sensitive indices of the rate of bone turnover (16-18). The aim of the present work was to examine the levels of MMP-9 in patients with MM and age-matched controls, and correlate them to the severity of disease, as well as to the rate of bone turnover. For this purpose, we measured MMP-9 levels and bone resorption biochemical indi- 
ces in our patients, and correlated them with the stage of their disease.

\section{Materials and methods}

\section{Patients}

The study population included 37 newly diagnosed patients with MM: nine in stage I, 12 in stage II and 16 in stage III according to Durie-Salmon classification (19). Serum samples from 12 persons, age- and sex-matched healthy volunteers, were used as controls. Informed consent for the study was obtained from all subjects.

The median age of the patient group was 63 years (range 39-84 years). There were 17 males (age 39-81 years) and 20 females (age 39-81 years). Monoclonal immunoglobulins $\lg \mathrm{G}, \lg \mathrm{A}$ and light chain disease were found in 22, 11 and four patients, respectively. No patient had received chemotherapy or radiotherapy prior to initial sampling. None of the patients had evidence of thyroid or parathyroid hormonerelated abnormalities or had received any therapy known to affect bone metabolism. According to standard X-ray evaluation of the patients, bone involvement was graded into four scores: no lesions (score 0 ), one bone involved or diffuse osteoporosis (score 1), more than one but less than four bone lesions (score 2) and more than four bone lesions or bone fracture present (score 3). Using these definitions, 11 patients had score 0 , four had score 1 , six had score 2, and 16 patients had score 3 .

\section{Methods}

Serum samples were collected from patients and controls, aliquoted into separate vials and stored at $-70^{\circ} \mathrm{C}$ after collection, and assayed at the end of the study, in order to avoid inter-assay variability.

Urinary N-telopeptide (NTx) cross-links of type I collagen were measured by a competitive-inhibition enzyme-linked immunosorbent assay (ELISA) (Osteomark Ostex International, Seattle, WA, USA), using a monoclonal antibody for NTx labeled with horseradish peroxidase. Results were collected for 24-h urinary concentration of creatinine and expressed as nanomoles of bone collagen equivalent per millimole of creatinine ( $\mathrm{nmol} \mathrm{BCE} / \mathrm{mM}$ creat). Free urine pyridinolines (F-Pyd) were measured by a competitive enzyme immunoassay (METRA PYD EIA assay, Metra biosystems, Mountain View, CA, USA) using monoclonal antibodies. Free urine deoxy-pyridinolines (F-Dpd) alone were measured by
METRA DPD EIA assay (Metra biosystems). Results for F-Pyd and F-Dpd alone were corrected for 24-h urinary concentration of creatinine and expressed as nanomoles per millimole of creatinine.

Measurements of IL- 6 and MMP-9 in serum samples collected from patients and controls were performed by solidphase sandwich ELISA with commercially available test kits, Quantikine ${ }^{\circledR}$ Human MMP-9 (total) and IL-6 (R\&D Systems Inc., Minneapolis, MN, USA).

Bone marrow cellularity and percentage of bone marrow infiltration by myeloma cells was estimated by bone marrow biopsies that were taken during local anesthesia from a point below the iliac crest.

\section{Statistical analysis}

Results are expressed as mean \pm SD. The non-parametric Kruskal-Wallis test and one-way analysis of variance (ANOVA) were assessed to test the existence of differences between different stages. The Student-Newman-Keuls test was used for pairwise comparison of subgroups. Statistical comparisons between the MM group and the control group were made using the non-parametric Mann-Whitney test. Correlations between the various measured parameters were calculated by Spearman's rank correlation coefficient. $p$-Values $<0.05$ were considered to be statistically significant.

\section{Results}

The mean concentration of IL- 6 in the entire group of patients was $6.69 \pm 5.51 \mathrm{pg} / \mathrm{mL}$ and was significantly higher in comparison to that found in the control group $(p<0.001)$ (Table 1). The mean values for infiltration and IL-6 in the group of MM patients were significantly higher with increasing stage of disease $(p<0.001, p<0.01$, respectively) (Table 1). Subsequently, we evaluated the measured parameters of disease activity, infiltration and IL- 6 according to the grade of bone disease. Bone marrow infiltration was significantly higher in grade 3 than in grade 0 $(51.88 \pm 21.28$ vs. $28 \pm 16.28, p=0.02)$. In contrast, mean serum levels of IL-6 were higher with advancing bone disease, but without statistical significance.

The mean concentrations of NTx, F-Pyd and F-Dpd were significantly higher in $\mathrm{MM}$ patients in comparison to those found in the group of controls $(p<0.001$

Table 1 Values of plasma cell infiltration, IL-6, MMP-9, and of biochemical markers of bone resorption in 37 untreated MM patients according to disease stage, and in controls. With the exception of MMP-9, values of all measured parameters were significantly higher in MM patients than in controls. Values for infiltration, IL-6, and urinary N-telopeptide (NTx) were significantly higher with advancing stage of the disease.

\begin{tabular}{lllll}
\hline & \multicolumn{2}{l}{ Multiple myeloma group } & Controls \\
\cline { 2 - 4 } & $\begin{array}{l}\text { Stage I } \\
\text { Mean ( } \pm \text { SD) }\end{array}$ & $\begin{array}{l}\text { Stage II } \\
\text { Mean ( } \pm \text { SD) }\end{array}$ & $\begin{array}{l}\text { Stage III } \\
\text { Mean }( \pm S D)\end{array}$ \\
\hline Infiltration & $22.56 \pm 16.09$ & $33.58 \pm 19.78$ & $56.25 \pm 16.78^{*}$ & $0.98 \pm 0.64^{+}$ \\
IL-6, pg/mL & $3.06 \pm 1.33$ & $5.71 \pm 3.48$ & $9.47 \pm 6.79^{* *}$ & $289.8 \pm 110.46^{+}$ \\
MMP-9, ng/mL & $59.25 \pm 33.09$ & $188.78 \pm 91.27^{* * *}$ & $131.2 \pm 96.32$ & $32.67 \pm 3.11^{+}$ \\
NTx & $86 \pm 67.49$ & $159 \pm 61.23$ & $222.4 \pm 47.68^{*}$ & $20.83 \pm 2.62^{*}$ \\
F-Pyd & $26.6 \pm 6.5$ & $54.3 \pm 19.6^{* *}$ & $58.4 \pm 23.8^{*}$ & $4.17 \pm 0.72^{+}$ \\
F-Dpd & $5.7 \pm 1.6$ & $12.3 \pm 5.3^{* * *}$ & $11.9 \pm 5.3^{* * *}$ & \\
\hline
\end{tabular}

*Stage III vs. I and II p $<0.001,{ }^{* *}$ Stage III vs. I $p<0.002,{ }^{* *}$ Stage II vs. I $p<0.004,{ }^{+}$control group vs. multiple myeloma group $\mathrm{p}<0.001$. 


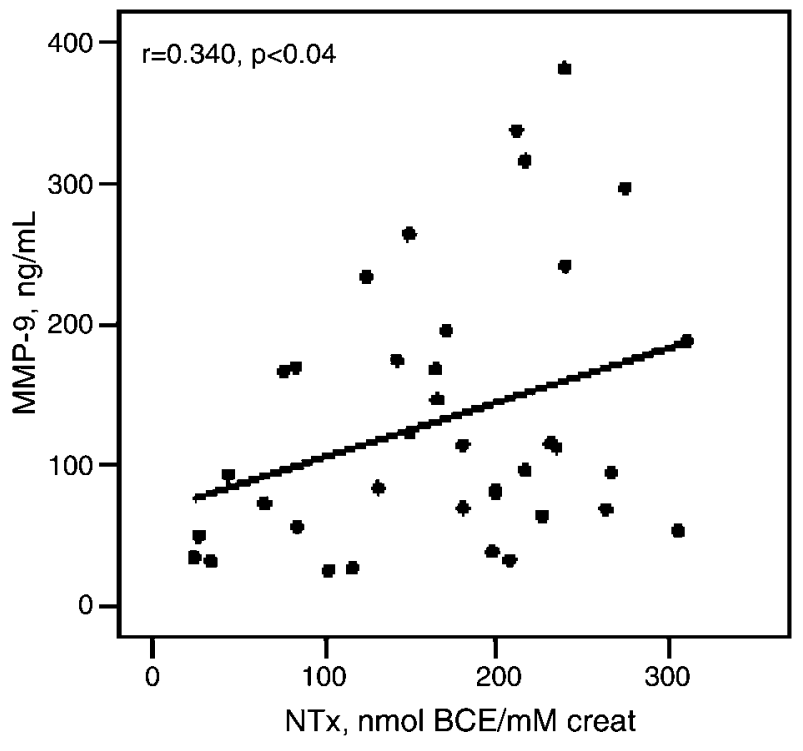

Figure 1 Correlation between serum MMP-9 levels and urinary $\mathrm{N}$-telopeptide (NTx) values in 37 newly diagnosed multiple myeloma patients. A significant positive correlation was found between serum MMP-9 and NTx values (Spearman's correlation coefficient, $r=0.340, p<0.04$ ).

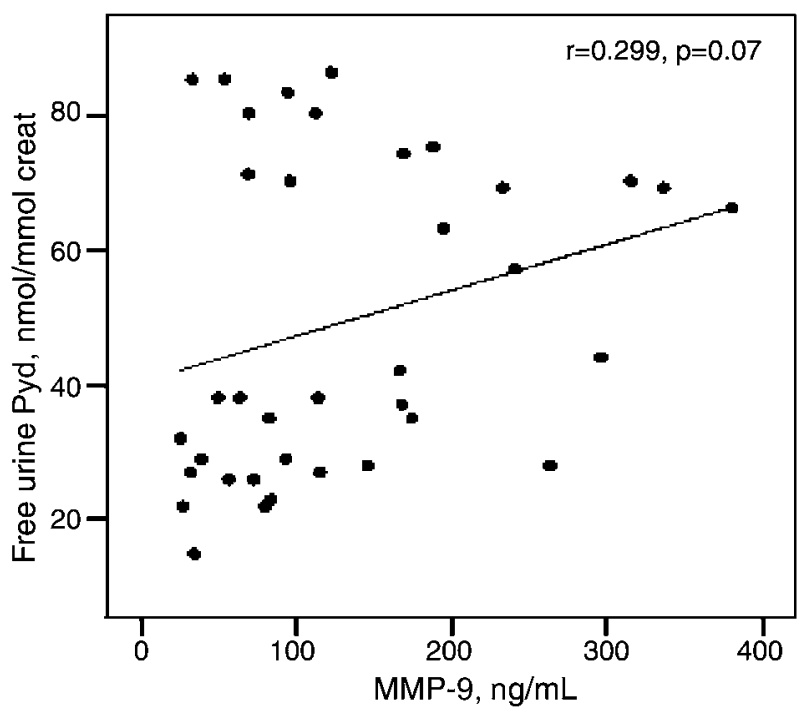

Figure 2 Serum MMP-9 levels and free urine pyridinoline (F-Pyd) values in 37 newly diagnosed multiple myeloma patients. There was a positive correlation between serum MMP-9 and F-Pyd values, which did not reach statistical significance (Spearman's correlation coefficient, $r=0.299$, $p=0.07)$.

in all cases) (Table 1). In the group of MM patients, the mean concentrations of NTx, F-Pyd and F-Dpd were significantly higher as the stage of disease advanced $(p<0.001, p<0.001, p<0.003$, respectively).

The biochemical parameters were analyzed according to the severity of bone disease at diagnosis, as estimated by radiographs of the whole skeleton (grade 0 to 3). Patients with progressive bone disease had elevated levels of F-Pyd and F-Dpd in comparison to patients without osteolytic lesions. More specifically, the mean concentrations of F-Pyd and F-Dpd were higher in grade 3 compared to grade 0 $(61.81 \pm 22.17$ vs. $30.55 \pm 15.91, p<0.002$, and $13.13 \pm$ 5.46 vs. $6.27 \pm 2.72, p<0.003$, respectively). In contrast, the mean concentrations of F-Pyd and F-Dpd in grades 0,1 and 2 were increasing gradually with advancing bone disease, but differences did not reach statistical significance. Moreover, mean concentrations of NTx were higher in grade 3 compared to grades 0,1 and 2 ( $p<0.001$ in all cases).

We further evaluated the levels of MMP-9 in healthy controls and in the group of patients. The mean serum concentration of MMP-9 $(132.37 \pm 95.14 \mathrm{ng} / \mathrm{mL})$ was lower in the group of patients compared to the control group $(289.80 \pm 110.46 \mathrm{ng} / \mathrm{mL}, \quad \mathrm{p}<0.001)$ (Table 1). Furthermore, MMP-9 mean serum concentration was significantly higher in stage II, in comparison to stage I $(p<0.004)$. Stratification of MMP-9 serum levels according to the grade of bone disease revealed higher levels in grade 2 vs. grades 0 and 1 ( $p<0.003, p<0.02$, respectively). Of note, a positive correlation was found between infiltration and NTx levels $(r=0.340, p<0.03)$ as well as between serum IL-6 and NTx levels $(r=0.340, p<0.04)$. A significant correlation was found between serum MMP-9 and NTx values $(r=0.340, p<0.04)$ (Figure 1). In addition, a trend was detected between MMP-9 values and F-Pyd and F-Dpd, which approached statistical significance $(r=0.299, p=0.07$ and $r=0.228, p=0.08$, respectively) (Figures 2 and 3 ).

\section{Discussion}

We and others have shown that there is an enhancement of osteoclastic activity in MM patients $(3,4,20)$. This process is mediated by factors produced locally by malignant and non-malignant cells in the microenvironment of bones. We have found that MMP-9

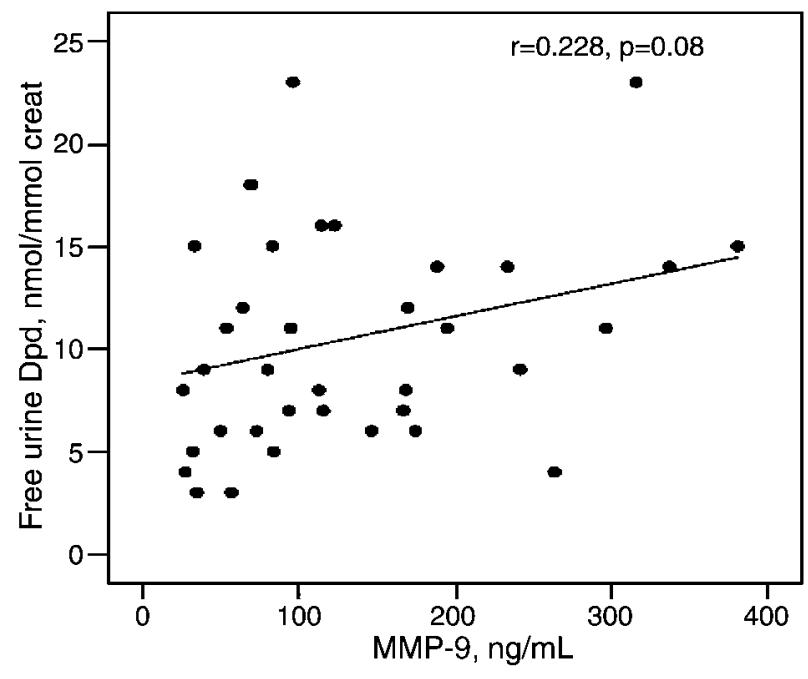

Figure 3 Serum MMP-9 levels and free urine deoxy-pyridinoline (F-Dpd) values in 37 newly diagnosed multiple myeloma patients. There was a positive correlation between serum MMP-9 and F-Dpd values, which did not reach statistical significance (Spearman's correlation coefficient, $r=0.228, p=0.08$ ). 
may play an important role in worsening bone disease in patients with MM.

In the present study, serum levels of MMP-9 in stages II and III MM patients were higher than in stage I patients, but serum MMP-9 levels in the whole patient group were lower compared to the age-matched control group. The explanation for this discrepancy remains unclear. In MM patients, reduced MMP-9 levels have been detected in tumors with elevated levels of syndecan-1 (21), however, decreased MMP-9 does not result from association with syndecan-1 expression in malignant cells (22). Lower MMP-9 levels in myeloma patients may be due to a reduction in MMP9 production from normal sources in MM, which leads to a net decrease in serum MMP-9 (21). It has been postulated that the predominant sources of serum MMP-9 activity in normal people are the circulating lymphocytes, neutrophils and monocytes in which MMP-9 is thought to play an important physiological role (23). As such cells may be crowded by malignant plasma cells in MM, future relevant studies of MMP-9 levels from the bone lesions may help clarify this issue. From this point of view, mast cells may also be important as they have been reported to produce MMP-9 (24), and mast cell chymase activates proMMP-9 (25). Mast cell release of MMP-9 and other lytic enzymes has been associated with tumor progression and aggressiveness (26).

The progression of the disease in MM patients is accompanied by an accelerated angiogenesis and production of proteolytic enzymes $(6,27)$. Furthermore, the growth of myeloma cells is regulated by a cytokine network in which IL-6 represents a major growth player. We found higher serum MMP-9 levels in patients with bone disease scores 2 and 3, in comparison to scores 0 and 1 . This observation suggests that the excessive MMP-9 activity in bone marrow plays a functional role in pathological conditions associated with $\mathrm{MM}$, such as tumor progression and osteolysis.

We found that the levels of serum MMP-9 correlated significantly to the estimated rate of bone turnover. Indeed, we report a positive correlation between urinary levels of NTx, MMP-9 $(r=0.328, p<0.04)$ and IL-6 $(r=0.340, p<0.04)$. Based on this finding, we and others postulate that the accelerated bone turnover is a result of locally produced factors (including MMP-9) in the microenvironment of the bone, which either accelerate bone turnover (3) or enhance their biological effect via MMP-9. In fact, our results show a trend for direct association of MMP-9 values with F-Pyd and F-Dpd suggesting the possible role of MMP-9, produced locally by myeloma cells, in the promotion of osteolytic bone disease. Published reports suggest that MMP-9 plays an important role in bone resorption $(7,16)$, and that MMP-9 production by plasma cells accelerates invasiveness and angiogenesis (28). The latter is related to the proliferative activity of malignant plasma cells, according to our previous results (29).

In the present study, serum levels of IL-6 correlated well with urinary levels of NTx, and also, in contrast with MMP-9, with disease stage, being significantly higher in $\mathrm{MM}$ patients than in controls, confirming previous results (29). Measurement, thus, of IL-6 may provide a useful marker for monitoring disease activity in MM. Interestingly, serum IL-6 levels have been reported to correlate well with bone involvement in systemic mastocytosis patients (30).

We have found that the mean concentrations of NTx, F-Pyd and F-Dpd were significantly higher in the MM patients compared to age-matched healthy controls $(p<0.001)$. Moreover, in the group of MM patients the mean concentrations of NTx, F-Pyd and F-Dpd were increasing significantly as the disease progressed $\quad(p<0.001, \quad p<0.001, \quad p<0.003$, respectively).

Finally, biochemical parameters were found to correlate to the severity of bone disease at diagnosis, as estimated by $\mathrm{X}$-rays. Thus, patients with progressive bone disease had elevated levels of F-Pyd and F-Dpd compared to patients without osteolytic lesions. Specifically, mean concentrations of F-Pyd and F-Dpd were higher in grade 3 than in grade $0(61.81 \pm 22.17$ vs. $30.55 \pm 15.91, \mathrm{p}<0.002$, and $13.13 \pm 5.46$ vs. $6.27 \pm 2.72, \mathrm{p}<0.003$, respectively).

In conclusion, we have found that levels of MMP-9 and of IL- 6 in the circulation of patients with MM correlate with both the radiological disease score and the rate of bone turnover as judged by biochemical markers of bone resorption and formation. Based on these findings, we propose measuring the levels of MMP-9 in the blood of patients with MM, since this determination may add information regarding the severity of their disease and the degree of its effect on bone well being.

\section{References}

1. Bataille R, Chappard D, Basle M. Excessive bone resorption in human plasmacytomas: direct induction by tumour cells in vivo. Br J Haematol 1995;90:721-4.

2. Alexandrakis MG, Passam FH, Malliaraki N, Katachanakis C, Kyriakou DS, Margioris AN. Evaluation of bone disease in multiple myeloma: a correlation between biochemical markers of bone metabolism and other clinical parameters in untreated multiple myeloma patients. Clin Chim Acta 2002;325:51-7.

3. Mundy GR. Myeloma bone disease. Eur J Cancer 1998; 34:246-51.

4. Alexandrakis MG, Kyriakou DS, Passam FH, Malliaraki N, Vlachonikolis IG, Karkavitsas N. Urinary N-telopeptide levels in multiple myeloma patients, correlation with Tc-99m-sestaMIBI scintigraphy and other biochemical markers of disease activity. Hematol Oncol 2003; 21:17-24.

5. Ray JM, Stetler-Stevenson WG. The role of matrix metalloproteases and their inhibitors in tumour invasion, metastasis and angiogenesis. Eur Respir J 1994; 7:2062-72.

6. Vacca A, Ribatti D, Presta M, Minischetti M, Iurlaro M, Ria $R$, et al. Bone marrow neovascularization, plasma cell angiogenic potential, and matrix metalloproteinase-2 secretion parallel progression of human multiple myeloma. Blood 1999;93:3064-73. 
7. Barille S, Akhoundi C, Collette M, Mellerin MP, Rapp MJ, Harousseau JL, et al. Metalloproteinases in multiple myeloma: production of matrix metalloproteinase-9 (MMP-9), activation of proMMP-2, and induction of MMP-1 by myeloma cells. Blood 1997;90:1649-55.

8. Mignatti $P$, Rifkin DB. Biology and biochemistry of proteinases in tumor invasion. Physiol Rev 1993;73:161-95.

9. Stetler-Stevenson WG, Aznavoorian S, Liotta LA. Tumor cell interactions with the extracellular matrix during invasion and metastasis. Annu Rev Cell Biol 1993; 9:541-73.

10. Niesvizky R, Warrell RP Jr. Pathophysiology and management of bone disease in multiple myeloma. Cancer Invest 1997;15:85-90.

11. Kelly T, Borset M, Abe E, Gaddy-Kurten D, Sanderson RD. Matrix metalloproteinases in multiple myeloma. Leuk Lymphoma 2000;37:273-81.

12. Van Valckenborgh E, Bakkus M, Munaut C, Noel A, St Pierre $\mathrm{Y}$, Asosingh K, et al. Upregulation of matrix metalloproteinase-9 in murine 5T33 multiple myeloma cells by interaction with bone marrow endothelial cells. Int $\mathrm{J}$ Cancer 2002;101:512-8.

13. Vande Broek I, Asosingh K, Allegaert V, Leleu X, Facon $\mathrm{T}$, Vanderkerken $\mathrm{K}$, et al. Bone marrow endothelial cells increase the invasiveness of human multiple myeloma cells through upregulation of MMP-9: evidence for a role of hepatocyte growth factor. Leukemia 2004;18:976-82.

14. Westermarck J, Kahari VM. Regulation of matrix metalloproteinase expression in tumor invasion. FASEB $\mathrm{J}$ 1999;13:781-92.

15. Derksen PW, de Gorter DJ, Meijer HP, Bende RJ, van Dijk M, Lokhorst HM, et al. The hepatocyte growth factor/Met pathway controls proliferation and apoptosis in multiple myeloma. Leukemia 2003;17:764-74.

16. Abildgaard N, Glerup H, Rungby J, Bendix-Hansen K, Kassem M, Brixen K, et al. Biochemical markers of bone metabolism reflect osteoclastic and osteoblastic activity in multiple myeloma. Eur J Haematol 2000;64:121-9.

17. Corso A, Arcaini L, Mangiacavalli S, Astori C, Orlandi E, Lorenzi A, et al. Biochemical markers of bone disease in asymptomatic early stage multiple myeloma. A study on their role in identifying high risk patients. Haematologica 2001;86:394-8.

18. Fonseca R, Trendle MC, Leong T, Kyle RA, Oken MM, Kay NE, et al. Prognostic value of serum markers of bone metabolism in untreated multiple myeloma patients. $\mathrm{Br}$ J Haematol 2000;109:24-9.
19. Durie BG, Salmon SE. A clinical staging system for multiple myeloma. Correlation of measured myeloma cell mass with presenting clinical features, response to treatment, and survival. Cancer 1975;36:842-54.

20. Anderson KC, Lust JA. Role of cytokines in multiple myeIoma. Semin Hematol 1999;36(1 Suppl 3):14-20.

21. Dhodapkar MV, Kelly T, Theus A, Athota AB, Barlogie B, Sanderson RD. Elevated levels of shed syndecan-1 correlate with tumour mass and decreased matrix metalloproteinase- 9 activity in the serum of patients with multiple myeloma. Br J Haematol 1997;99:368-71.

22. Kaushal GP, Xiong $X$, Athota AB, Rozypal TL, Sanderson RD, Kelly T. Syndecan-1 expression suppresses the level of myeloma matrix metalloproteinase-9. Br J Haematol 1999;104:365-73.

23. Stetler-Stevenson WG, Liotta LA, Kleiner DE Jr. Extracellular matrix 6: role of matrix metalloproteinases in tumor invasion and metastasis. FASEB J 1993;7:143441.

24. Tanaka A, Matsuda H. IgE crosslinkage of Fcepsilon receptor $\mathrm{I}$ induces both production and activation of matrix metalloproteinase- 9 in mast cells. Cell Immunol 2004;228:66-75.

25. Tchougounova E, Lundequist A, Fajardo I, Winberg JO, Abrink M, Pejler G. A key role for mast cell chymase in the activation of pro-matrix metalloprotease- 9 and promatrix metalloprotease-2. J Biol Chem 2005;280:9291-6.

26. Theoharides TC, Conti P. Mast cells: the Jekyll and Hyde of tumor growth. Trends Immunol 2004;25:235-41.

27. Alexandrakis MG, Passam FH, Dambaki C, Pappa CA, Stathopoulos EN. The relation between bone marrow angiogenesis and the proliferation index Ki-67 in multiple myeloma. J Clin Pathol 2004;57:856-60.

28. Vacca A, Ribatti D, Roccaro AM, Ria R, Palermo L, Dammacco $F$. Bone marrow angiogenesis and plasma cell angiogenic and invasive potential in patients with active multiple myeloma. Acta Haematol 2001;106:162-9.

29. Alexandrakis MG, Passam FH, Pappa CA, Dambaki C, Sfakiotaki G, Alegakis AK, et al. Expression of proliferating cell nuclear antigen (PCNA) in multiple myeloma: its relationship to bone marrow microvessel density and other factors of disease activity. Int $\mathrm{J}$ Immunopathol Pharmacol 2004;17:49-56.

30. Theoharides TC, Boucher W, Spear K. Serum interleukin6 reflects disease severity and osteoporosis in mastocytosis patients. Int Arch Allergy Immunol 2002;128: 344-50.

Received May 12, 2005, accepted July 3, 2005 